\title{
Basic science to clinical trials in non-alcoholic fatty liver disease and alcohol-related liver disease: collaboration with industry
}

\author{
Amon Asgharpour ${ }^{\#}$, Amreen Dinani", Scott L. Friedman \\ Division of Liver Diseases, Icahn School of Medicine at Mount Sinai, New York, NY, USA \\ Contributions: (I) Conception and design: All authors; (II) Administrative support: None; (III) Provision of study materials or patients: None; (IV) \\ Collection and assembly of data: A Asgharpour, A Dinani; (V) Data analysis and interpretation: None; (VI) Manuscript writing: All authors; (VII) \\ Final approval of manuscript: All authors. \\ "These authors contributed equally to this work. \\ Correspondence to: Scott Friedman, MD. Division of Liver Diseases, Icahn School of Medicine at Mount Sinai, 1425 Madison Ave, Box 1123 , New \\ York, NY 10029, USA. Email: scott.friedman@mssm.edu.
}

\begin{abstract}
Non-alcoholic fatty liver disease (NAFLD) and alcohol-related liver disease (ALD) are highly prevalent forms of chronic liver diseases globally, associated with rising all-cause morbidity and mortality. While distinct diseases, NAFLD and ALD share several similarities; both can result in fatty liver disease, steatohepatitis, associated hepatic fibrosis and cirrhosis-related complications, including hepatocellular carcinoma (HCC). Our understanding of the pathophysiology and manifestations of these diseases has advanced significantly, which has established a new foundation to identify therapeutic targets and test new treatments. This review underscores emerging pathogenic pathways that establish a template for target identification and clinical trials. Success is critically dependent upon productive interactions between academic investigators and industry to address unmet therapeutic needs in NAFLD and ALD.
\end{abstract}

Keywords: Non-alcoholic fatty liver disease (NAFLD); non-alcoholic steatohepatitis; alcoholic liver disease; clinical trials

Received: 27 August 2019; Accepted: 17 January 2020; Published: 05 January 2021.

doi: $10.21037 /$ tgh.2020.01.04

View this article at: http://dx.doi.org/10.21037/tgh.2020.01.04

\section{Introduction}

Non-alcoholic fatty liver disease (NAFLD) (1) and alcoholrelated liver disease (ALD) have a rising prevalence worldwide $(1,2)$; both can lead to similar complications including end-stage liver disease, increased risk of cardiovascular disease (3), and hepatocellular carcinoma (HCC) $(3,4)$. Together, they are the most common indication liver transplantation $(5,6)$. While the precipitating factors for NAFLD and ALD are different, there is significant overlap between these disease (7-9), and when they co-exist the progression of disease is accelerated. Both disorders share common pathways resulting in hepatic steatosis, steatohepatitis ( $\mathrm{SH}$ ) and activation of inflammatory and fibrogenic pathways. In this review we define emerging pathogenic pathways and their translation to new therapeutic targets that are being evaluated in clinical trials. Success is critically dependent upon productive interactions between academic investigators and industry to address unmet therapeutic needs in NAFLD and ALD.

Why is there so much interest in nonalcoholic steatohepatitis?

The NAFLD and obesity epidemics are rising and inextricably linked. NAFLD, comprising both nonalcoholic fatty liver (1) and non-alcoholic steatohepatitis is present approximately 65-90 million adults in the United States (10) (20-30\% of the population) and is recognized as a leading cause of HCC (11), and is likely to become the most common indication for liver transplantation in the coming years (12). Nonalcoholic steatohepatitis (NASH), characterized by inflammation and hepatocyte 
damage (11) is the progressive form of the disease, typically associated with fibrosis and cirrhosis. NASH accounts for $20-30 \%$ of the NAFLD population of which $4-6 \%$ can progress to cirrhosis, but this percentage is likely an underestimate because the disease is not easily diagnosed, typically asymptomatic, and up to $30 \%$ of affected patients have normal liver enzymes. The prevalence of NASH in the United States is projected to grow from 20\% to $27 \%$ from 2015 to 2030 . Furthermore, 20\% of these cases will have advanced fibrosis or cirrhosis (F3/F4), associated with accelerated disease (13), increased risk of liver and non-liver related mortality $(14,15)$ and rising economic burden (16).

The development of highly efficacious direct acting antiviral agents that cure $>95 \%$ of chronic hepatitis C (CHC), coupled with aggressive efforts to eliminate hepatitis C by 2030 (16), have led to a dramatic refocusing of drug development efforts towards treatments for NASH and associated fibrosis. There are over 100 registered clinical trials for NASH in different phases of development. Both pharmaceutical and biotech companies with successful histories of drug development for viral hepatitis have shifted their focus towards NASH, while those companies with programs in cardiometabolic disease are leveraging the interconnection between NASH, obesity and type 2 diabetes (T2DM). Because there is a strong association between the stage of fibrosis and both liver and all-cause morbidity and mortality, anti-fibrotic agents independent of etiology are also rapidly emerging, with the goal of halting fibrosis progression to prevent the development of cirrhosis and its complications. The surge in prevalence of NAFLD has precipitated an urgent interest by clinicians, basic and translational investigators to work closely with industry in order to increase disease awareness, refine diagnostics, optimize clinical care pathways and, most importantly, address the unmet need to develop therapeutic targets that ameliorate or steatohepatitis and fibrosis.

\section{Why is there a beightened focus on alcohol-related liver disease?}

ALD encompasses a spectrum of alcohol-related diseases that includes alcoholic steatosis, alcoholic steatohepatitis (ASH) and advanced ALD characterized by alcoholic hepatitis (AH), cirrhosis and consequent complications (hepatic encephalopathy, jaundice, portal hypertension related complications and HCC). Because its clinical presentation can vary greatly, the true disease burden of ALD is likely to be underrepresented by current prevalence estimates. ALD is typically under-reported by patients because of the associated social stigma, and is often undiagnosed as a co-morbid etiology in conjunction with other chronic liver diseases. With that in mind, ALD accounts for up to $48 \%$ of cirrhosis-related deaths in the United States (17). Alcohol-related mortality remains the fourth leading preventable cause of death in the United States (17). In a 2016 report, ALD was the leading indication for liver transplantation (18), and a 2018 US-population-based study demonstrated increasing alcoholic cirrhosis-related deaths in individuals aged 25 to 34 years (3). In a recent analysis of the National Health and Nutrition Examination Survey (NHANES) database from 2001 to 2016, there was a stable prevalence of ALD of $4.3 \%$ (95\% CI, $3.5-5.0 \%$ ) to $4.7 \%$ (95\% CI, $4.2-5.1 \%$ ) $(\mathrm{P}=0.69)$, but increased prevalence of those with stage two fibrosis, from $0.6 \%(95 \% \mathrm{CI}, 0.5-0.8 \%)$ to $1.5 \%(95 \% \mathrm{CI}$, $1.3-1.8 \%)(\mathrm{P}<0.001)$, and those with stage 3 fibrosis or higher, from $0.1 \%$ (95\% CI, $0.02-0.10 \%)$ to $0.2 \%$ (95\% CI, $0.2-0.4 \%)(\mathrm{P}=0.045)$ portending a growing burden of ALD in the near future (19).

Although ALD has been recognize for centuries, therapies remain outdated and inadequate, with current recommendations largely based on studies performed decades ago. Alcohol abstinence remains the most effective therapy to reverse ALD (20,21). Early liver transplantation has been effective in selected patients with acute $\mathrm{AH}$ with both short and long-term mortality benefits $(22,23)$, but this is not feasible for the large majority of ALD patients. As a result, there is a growing interest from regulatory agencies, industry and clinicians to develop effective pharmacotherapies for ALD, in combination with community-based approaches to reduce alcohol-dependency and associated morbidities.

\section{What have we learned so far?}

NASH has a highly variable clinical course, with some patients demonstrating spontaneous regression while others progressing inexorably to advanced fibrosis. As a result, designing clinical trials is challenging. The inclusion criteria for NASH clinical trials has evolved from scoring systems that quantify pathologic elements of the disease, towards assessments that rely on evidence of steatohepatitis resolution and/or improvement in fibrosis. Liver biopsy remains the gold standard for diagnosing NASH despite its limitations, invasive nature, and sampling variability. Clinical proof-of-concept studies can increasingly define 
NASH using surrogate markers (e.g., FIB-4, NAFLD fibrosis score), combined with risk factors that increase the likelihood of having NASH (e.g., type 2 diabetes and obesity) and/or imaging technologies including vibration controlled transient elastography (VCTE) to assess liver stiffness and hepatic steatosis, magnetic resonance imaging proton density fat fraction (MRI-PDFF) to assess fat and multiparametric MRI to detect changes associated with inflammation and fibrosis. There is intense interest in developing additional surrogate endpoints to measure improvement in NASH and fibrosis (noninvasive serological biomarkers of fibrosis such as cytokeratin-18 (CK-18) and European Fibrosis Panel (ELF), among others. However, validating these non-invasive endpoints in order to supplant biopsy in Phase $2 \mathrm{~b}$ and 3 trials remains challenging, and these later-stage clinical trials still rely on histologic endpoints. There are ongoing efforts to standardize the definition and staging of NASH using both non-invasive and biopsy-based endpoints. Achieving histological improvement in Phase 2a trials is difficult because these studies are more commonly short term (e.g., 12-16 weeks). As a result, efficacy predicted using non-invasive surrogates does not always translate into biopsy-based improvements in longer-term trials.

Ultimately, the effectiveness of NASH therapies will be measured by the impact on liver-and all-cause mortality, which is primarily driven by presence of advanced fibrosis and cirrhosis. Detecting a 1-stage change in fibrosis may require up to 7 years. Thus, even if drugs are approved based on histologic improvement, long term success, and therefore long-term drug approval, will be defined by demonstrating improvement in how a patient feels, functions and/or survives. The evolving landscape for drug evaluation is captured by a recent Food and Drug Administration (FDA) guidance in NASH clinical trial design $(24,25)$.

A major obstacle in assessing effectiveness of NASH therapies has been the high placebo response in clinical trials, as well as unexpected negative results from potential therapies that showed promise in earlier stages of drug development (26). The placebo response varies depending on the surrogate endpoint being evaluated, but likely reflects in part the impact of inadvertent lifestyle changes among placebo recipients. In a recent meta-analysis of 39 clinical trials, placebo was associated with 2 or more points improvement in NAS in $25 \%$ (95\% CI, $21-29 \%)$ of patients, with similar results in an analysis restricted to patients with no worsening in fibrosis. Similarly, $30 \%$ of placebo-treated patients had at least 1-point improvement in individual components of NAS and fibrosis stage improved in $21 \%$ of placebo-treated patients (27). It is important to clarify the basis for the high placebo response when designing clinical trials by enriching for patients with more advanced disease using evolving non-invasive surrogates.

Much like in NAFLD, clinical trial design in ALD is challenging due to the variability of disease presentation and natural history, lack of non-invasive or serological testing for diagnosis and staging, and uneven patient follow-up. Clinical trials and approved therapies focus on $\mathrm{AH}$, a subset of ALD associated with significant morbidity and mortality. Liver transplantation and corticosteroids are the only approved therapies. The end-points achieved with corticosteroid therapy are typically short, (e.g., 28-day and 6-month mortality) $(28,29)(30)$, with variable long-term mortality benefit among corticosteroid recipients (31). The lack of standardized criteria for defining early and intermediate stages of ALD further compounds the difficulties in conducting clinical trials, as these are typically based on self-reporting of alcohol use. In contrast, clinical staging methods for advanced ALD with AH are better validated and may include specific quantitative variables. Regulatory authorities recommend utilizing a second prognostic score such as model for end-stage liver disease (MELD), agebilirubin-international normalized ratio-creatinine (ABIC), Glasgow score or Lille score. The inclusion of liver biopsy for AH studies is highly controversial, however biopsy can exclude diseases that mimic $\mathrm{AH}$ (e.g., sepsis), but make it more difficult to enroll patients in clinical trials because of its high risk in very ill or unstable patients. Importantly, placebo-controlled trials are necessary to evaluate the rigor of a therapy, but withholding a known effective therapy makes designing a placebo-controlled trial in ALD difficult and unethical; thus, currently most therapies for $\mathrm{AH}$ require a comparison to corticosteroids rather than placebo $(32,33)$.

\section{Therapeutic targets}

NASH and ALD are driven by different etiologies, but they share many clinical, pathological and histological features. Both entities involve altered lipid metabolism, hepatocyte apoptosis, activation of the innate immune system and hepatic stellate cell activation, however they are also distinguished by engagement of disease-specific molecular signaling pathways and dysregulation of unique microRNAs. 
Based on the pathophysiology of NASH, therapeutic targets are categorized into three groups: (I) antiinflammatory; (II) anti-steatotic; (III) anti-fibrotic. Several drugs in clinical trials display more than one of these three activities. Furthermore, there is increasing interest in leveraging anti-diabetic therapies for NASH given the coexistence of both conditions in many patients, and the likely contribution of T2DM to NASH pathogenesis. Because even those drugs that show benefit only improve NASH in a minority of patients, there is also increasing interest in combination therapies that target multiple drivers of NASH. Given the overlap between NASH and ASH, there is also is a growing interest in exploiting shared pathways to develop therapies for both diseases. In practical terms, most agents are being tested first in NASH patients, with the likelihood that those showing promise will be subsequently evaluated in ALD.

Here we highlight several molecular targets in later stages of clinical development, such as Phase 2 and 3 trials (Table 1).

\section{Peroxisome proliferator-activated receptors (PPARs)}

PPARs are ligand-activated transcription factors of nuclear hormone receptor superfamily comprising three subtypes: $\operatorname{PPAR} \alpha, \operatorname{PPAR} \gamma$, and PPAR $\beta / \delta$ (57). They play a crucial role in metabolic function and energy homeostasis; PPAR $\alpha$ reduces triglycerides and regulates energy homeostasis, PPAR $\gamma$ increases insulin sensitivity to promote glucose metabolism and PPAR $-\beta / \delta$ 's has a predominant role in fatty acid metabolism. Thiazolidinediones (TZDs) are PPAR- $\gamma$ ligands that are widely utilized in the management of T2DM and also yield improvements in steatosis, steatohepatitis and hepatic fibrosis $(58,59)$. PPAR- $\gamma$ ligands' efficacy is greater in patients with NASH and T2DM than in those with NASH without T2DM, which needs to be further explored (60). While TZDs are attractive NASH targets, their appeal is limited by side effects of weight gain, osteopenia, peripheral edema and fluid retention.

Elafibranor, a selective dual PPAR- $\alpha / \delta$ agonist, devoid of PPAR- $\gamma$ effects, is a promising metabolic target for NASH. It is liver-targeted with little or no activity in skeletal muscle; the PPAR $\alpha$ component improves insulin sensitivity and lipid homeostasis by increasing fatty acid oxidation and PPAR- $\delta$ exerts an anti-inflammatory effect. In a post-hoc analysis of a phase $2 \mathrm{~b}$ trial, the drug improved metabolic features of NASH and has a favorable cardiometabolic profile (34). It is currently being tested in phase 3 trial: RESOLVE IT (NCT02704403).

\section{Thyroid hormone receptor-beta agonist}

Thyroid hormone receptor (56) signaling regulates organogenesis, growth and differentiation, regulation of energy and metabolism, and lipid and glucose homeostasis. The THR $\alpha$ subunit is primarily expressed in the brain, skeletal and cardiac muscle, and is responsible for thyrotoxicosis effects, while the $\beta$ subunit is expressed in the liver, brain and kidneys, and predominantly responsible for metabolic regulation. Both subclinical hypothyroidism and hypothyroidism are more prevalent in NAFLD patients, (61-63) and the liver-focused effect of thyroid hormone receptor-beta (THR $\beta$ ) subunit makes THR $\beta$ agonists attractive therapeutic prospects for metabolic syndrome, NAFLD and even HCC (64). Human studies have shown that THR $\beta$ agonist activation can result in the breakdown of fatty acids and ameliorate mitochondrial function to reduce lipotoxicity to significantly reduce low-density lipoprotein (LDL), triglycerides (TG) and hepatic steatosis $(35,65,66)$.

MGL-3196 and VK2809, both selective THR $\beta$ agonist have been targeted in NASH specifically to focus on reducing injury due to lipids, or 'lipotoxicity'. MGL-3196 was studied in a 36-week randomized, double blind placebo controlled phase 2 trial in patients with NASH, with reduction in hepatic steatosis (HS) measured by MRI-PDFF as a primary endpoint and impact on NASH, liver enzymes, indirect fibrosis biomarkers and lipids as a secondary endpoints at weeks 12 and 36. There was a significant reduction in hepatic steatosis in patients treated with MGL3196 compared to placebo $36 \%$ at 12 weeks and $37 \%$ at 36 weeks with lower dose; $42 \%$ and $49 \%$ respectively with higher dose therapy, and $8 \%$ and $10 \%$ respectively with placebo). Secondary endpoints of improvement in lipid profile, liver enzymes and NASH histological endpoints were also achieved in the treatment arms compared to placebo. The greatest effect on NASH endpoints occurred in those patients who achieved a $\geq 30 \%$ fat reduction measured by MRI-PDFF (37\% in MRI-responders versus $4 \%$ in MRI-non-responders, $\mathrm{P}=0.001)(67,68)$.

VK2809, a selective THR $\beta$ agonist, like MGL-3196 is also being evaluated in NASH. In a 16-week Phase 2 randomized placebo-controlled trial, NAFLD patients were identified by MRI-PDFF $>8 \%$ and LDL-C $>110 \mathrm{mg} / \mathrm{dL}$, with a primary endpoint of change in LDL-C, secondary endpoints including a change in hepatic fat measured by MRI-PDFF and effects on other atherogenic lipoproteins. There was a significant reduction in placebo adjusted percentage change in LDL-C at week 12 in both VK2809 


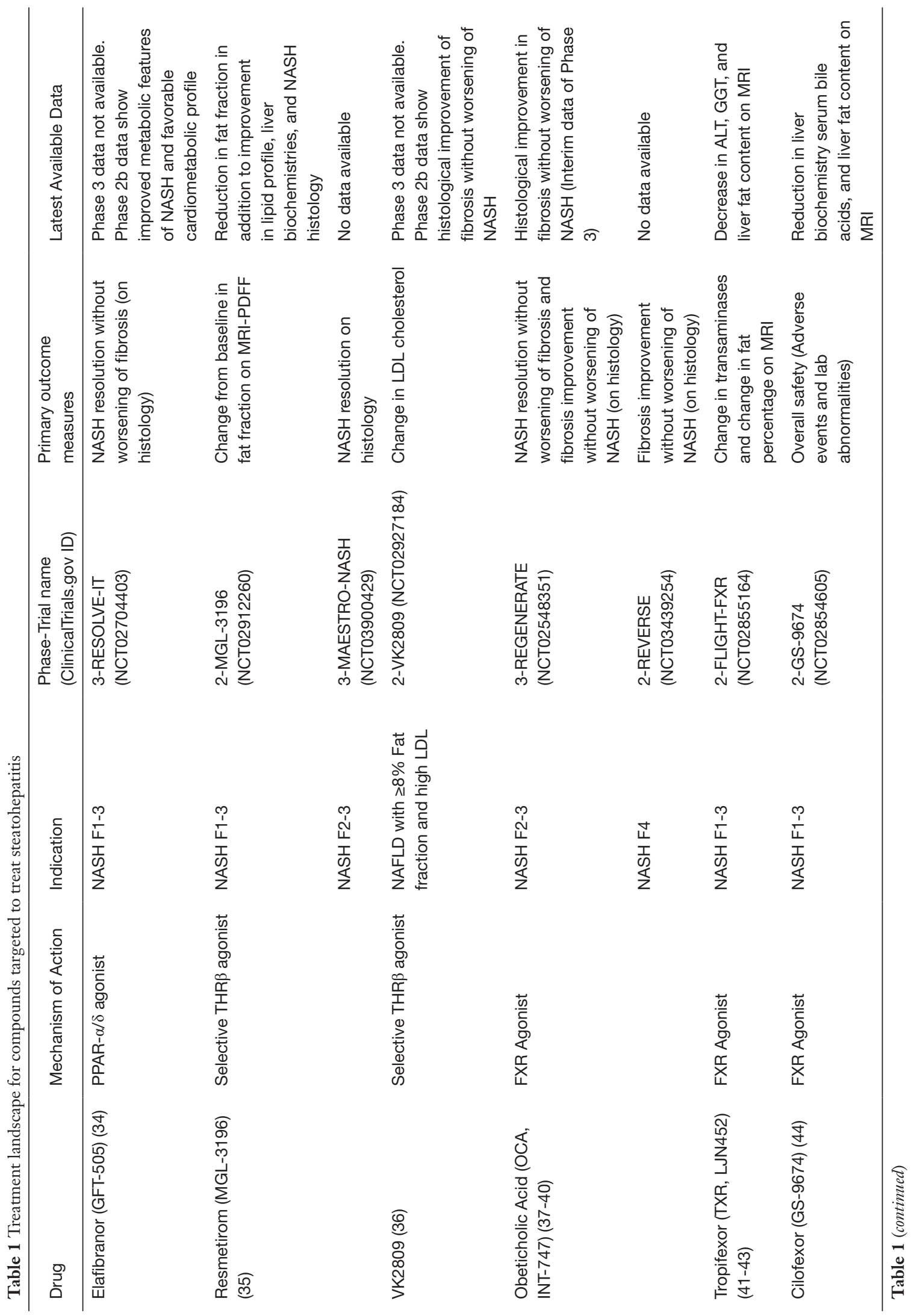




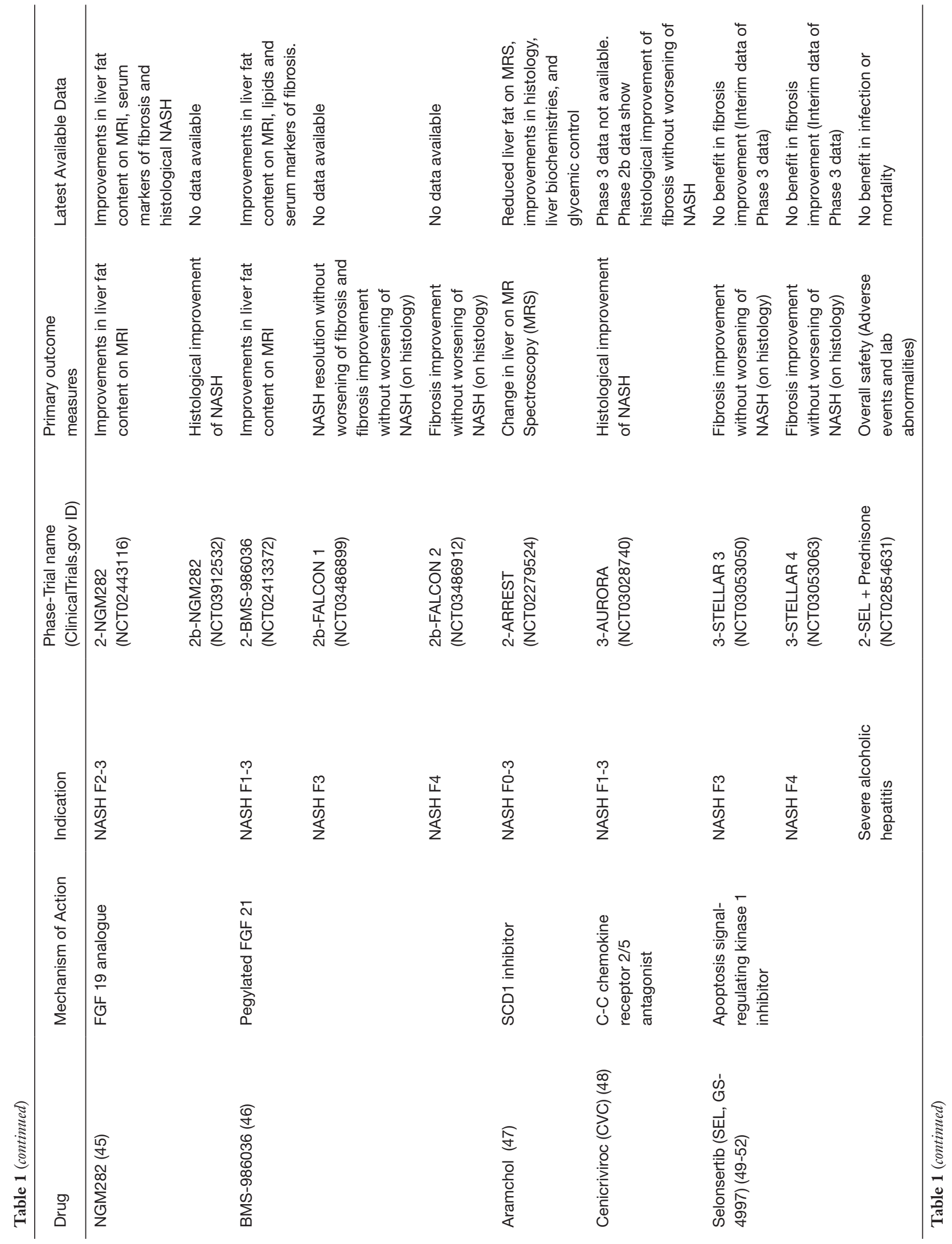




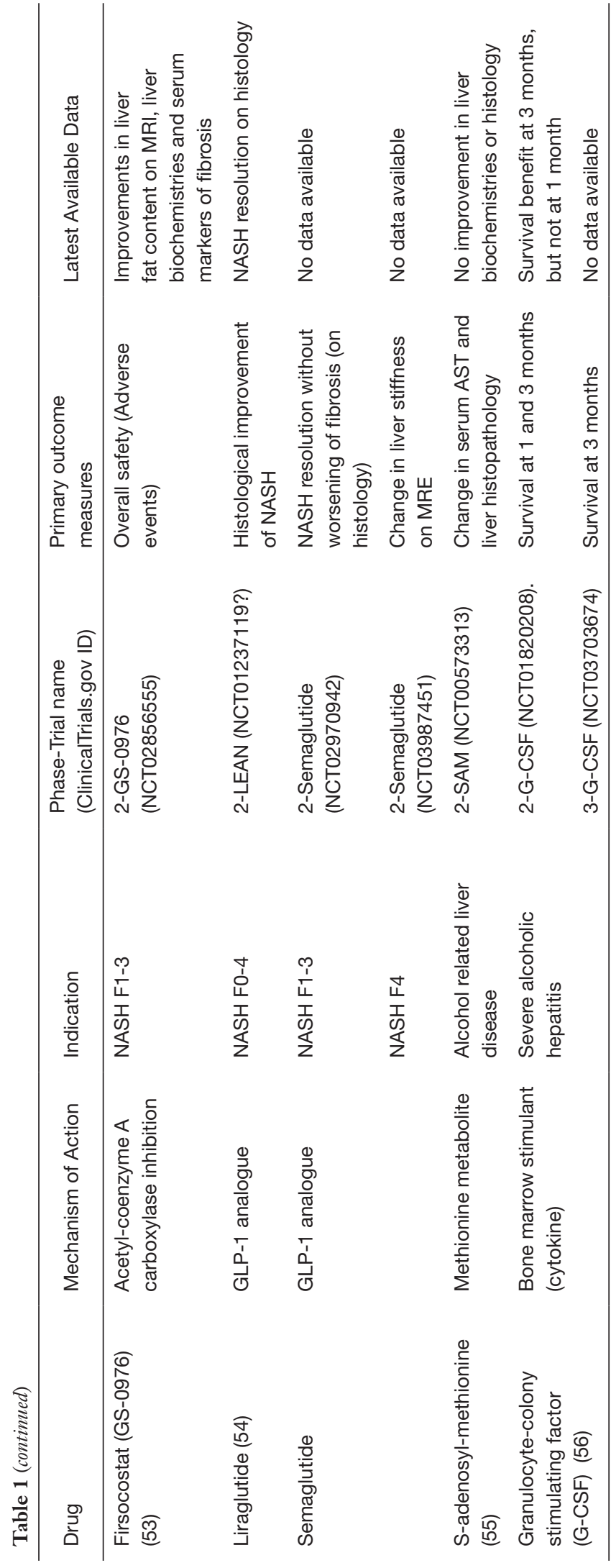

arms $(-23.6 \%$ in every other day arm and $-20.2 \%$ in the daily arm). VK2809 led to a relative reduction of $56.5 \%$, $59.7 \%$ in hepatic fat following either every-other-day or daily administration at week 12 compared to placebo (8.9\%). In addition, there were significant reductions in lipoprotein a and apolipoprotein B in both VK2809 arms compared to placebo (36). MGL-3196 is moving forward with phase 3 clinical trial (NCT03900429) and VK2809 is planned for a phase 2B study.

\section{Bile acids and their pathways}

Bile acids are synthesized from cholesterol in hepatocytes, then secreted into the bile canaliculi with other constituents of bile including water, cholesterol, bilirubin, phospholipids and inorganic salts. Bile acids have a well-established role in cholesterol homeostasis and lipid digestion via emulsification of lipids in the small intestine (69). In more recent years, nuclear (farnesoid $\mathrm{X}$ receptor, FXR), and transmembrane (Takeda G-protein coupled receptor clone 5, TGR5) receptors for bile acids have been discovered, greatly advancing our understanding of the endocrine and paracrine functions of bile acids (70). Bile acids are potent signaling molecules that play roles in glucose metabolism, lipid metabolism, inflammation and liver fibrosis. These emerging roles of bile acids as signaling molecules have been exploited for clinical use through the development of synthetic ligands that activate nuclear receptors, in particular the farnesoid X receptor (FXR)

Among FXR ligands, 6-ethyl chenodeoxycholic acid (6-ECDCA), more commonly known as obeticholic acid (OCA) or INT-747, is the best studied selective FXR agonist, with hepatoprotective and anti-cholestatic activity (71). OCA is a bile acid with 100-fold greater affinity for FXR than chenodeoxycholic, the natural ligand for FXR and has reduced TGR5 activity $(72,73)$. Early phase 2 human studies demonstrated improvements in insulin sensitivity, GGT, weight and increases in fibroblast growth factor 19 (FGF 19, a downstream target of FXR) after six weeks of treatment (37) with improvements in insulin sensitivity as the primary endpoint. Larger, longer studies reinforced its potential efficacy. In particular, the phase $2 \mathrm{~b}$ FLINT study, relied on liver biopsy with improvements in liver histology as the primary endpoint after 72 weeks of therapy (38). In the FLINT trial, OCA achieved greater NASH resolution and improvements in fibrosis in non-cirrhotic patients with NASH compared to placebo-treated patients. OCA treatment was associated with increases in total 
serum cholesterol and LDL cholesterol, a decrease in HDL cholesterol and more pruritus compared to patients receiving placebo (38). Subsequent studies have demonstrated that atorvastatin can mitigate the increase in LDL cholesterol in patients receiving OCA (39), and these medications were safe and well tolerated when administered together. OCA is currently being studied in a phase 3 trial in patients with NASH and stage 2-3 fibrosis (REGENERATE, NCT02548351) with histological and liver-related outcomes endpoints with an anticipated duration of 7 years. Interim analysis of OCA in the REGERNERATE trial after 18 months of treatment (40) reinforces OCA's antifibrotic properties, with greater fibrosis improvement compared to placebo $(23.1 \%$ with OCA $25 \mathrm{mg} ; 17.6 \%$ with $10 \mathrm{mg}$ OCA and $11.9 \%$ in placebo patients. However, there was no significant NASH resolution compared to placebo (40). There was also dose-dependent pruritus, which required drug discontinuation in $9 \%$ of patients. Studies testing OCA in patents with compensated NASH cirrhosis are ongoing (NCT03439254) utilizing a dose escalation approach. Vigilance is being exercised in patients with cirrhosis because of episodes of decompensation reported in patients with primary biliary cholangitis $(\mathrm{PBC})$ and cirrhosis that led to a black-box warning, although these patients were given doses in excess of those recommended in patients with cirrhosis.

Tropifexor (TXR), a non-bile acid with high affinity for the FXR receptor, can induce FXR target genes with no significant TGR5 activation (41). The compound is currently in phase 2 trials for NASH and $\mathrm{PBC}$ (NCT02855164 and NCT02516605 respectively). The phase 2 trial, FLIGHT-FXR, is ongoing and planned for 48 weeks, and interim analysis was presented in 2018 (26). Interim endpoints included ALT, GGT and liver fat content on MRI-PDFF after 12 weeks of treatment. TXR elicited pruritus at higher doses compared to placebo, with a dose related increase in LDL cholesterol and decrease in HDL cholesterol, similar to OCA's effects. At 12 weeks there was a relative decrease in liver fat content by MRI PDFF, with $-9.8 \%$ in placebo, $-16.9 \%$ with TXR $60 \mu \mathrm{g}$, and $-15.6 \%$ with TXR $90 \mu \mathrm{g}$. There was also a dose-related decrease in GGT and ALT (26). Further analysis demonstrated that TXR's effect was greater in patients with lower BMI, suggesting that a weight-based dosing approach may be necessary (43).

Cilofexor (GS-9674), a nonsteroidal, non-bile acid FXR agonist has been investigated in a 24-week phase 2 trial for patients with NASH and stage $1-3$ fibrosis. The initial findings demonstrated tolerability and no difference in lipids compared to placebo (44). Additionally, total bile acids and $\mathrm{C} 4$ were decreased, which are pharmacodynamic markers of FXR activation. More importantly, there was improvement in hepatic steatosis on MRI-PDFF that was dose dependent with $39 \%, 14 \%, 12.5 \%$ of patients achieving $\geq 30 \%$ decline in fat content for $100 \mathrm{mg}$ GS9674, $30 \mathrm{mg}$ GS-9674, and placebo, respectively. There was also a statistical improvement of GGT. While there was no difference in pruritus at the $30 \mathrm{mg}$ dose compared to placebo, there was more moderate to severe pruritus with the $100 \mathrm{mg}$ dose compared to placebo (44). Cilofexor has also shown promising efficacy in treating primary sclerosing cholangitis (PSC) with further planned for both NASH and PSC (74).

FXR activation stimulates increased FGF 19 secretion by the intestine, a key metabolic regulator that inhibits gluconeogenesis, regulates bile acid synthesis via CYP7A1 and promotes glycogen synthesis (75). NGM282 is an engineered analog of FGF 19 that is non-tumorigenic through deletion of the mitogenic region of the molecule. It inhibits de novo lipogenesis, improves insulin sensitivity, lowers liver transaminases, and demonstrated antifibrotic and anti-inflammatory properties in a murine model of NASH (76). This initial data led to a phase 2 human study testing a daily injection of NGM282 for 12-week with pre-and post-treatment liver biopsies, based and histologic improvement as a primary endpoint, compared to a historical placebo. There was a significant reduction in liver transaminases in both 1 and $2 \mathrm{mg}$ treatment groups within 2 weeks, which was sustained through the course of treatment. Histologic improvements were seen with $3 \mathrm{mg}$ NGM282 daily compared to historic controls in steatosis ( $74 \%$ versus $33 \%$ ), inflammation ( $42 \%$ versus $32 \%$ ), ballooning ( $53 \%$ versus $30 \%$ ) and fibrosis ( $42 \%$ versus $21 \%)$. No major adverse events were reported, but there was an increase in serum cholesterol, and rosuvastatin was administered to counteract this effect (45). Further studies are underway in a phase $2 \mathrm{~b}$ study for 24 weeks with varying doses of NGM282 compared to placebo (NCT03912532).

\section{C-C chemokine receptor types 2 and 5 antagonism}

Inflammation in response to hepatic injury leads to activation of monocytes and macrophages, which produce inflammatory cytokines and chemokines that trigger hepatic stellate cell activation with fibrosis (77). Inhibiting this inflammatory, fibrogenic pathway was 
explored with cenicriviroc (CVC), a dual antagonist of $\mathrm{C}-\mathrm{C}$ chemokine receptor types 2 and 5. Murine NASH studies demonstrated decreased serum transaminases in mice treated with CVC at both $20 \mathrm{mg}$ and $100 \mathrm{mg}$ daily dosing versus control. Additionally, there was less collagen deposition in the liver in both CVC treatment groups compared to control with a lower NAS in both treatment groups compared to control (78). The phase $2 \mathrm{~b}$ clinical trial, CENTAUR, in patients with NASH and stage 1-3 fibrosis investigated CVC $150 \mathrm{mg}$ daily versus placebo for a year, with a primary endpoint of $\geq 2$-point improvement in NAS and no worsening of fibrosis at year 1 . While the primary endpoint was not met, there was an improvement in fibrosis in patients treated with CVC for 1 year: $20 \%$ of CVC patients had improvement in fibrosis without worsening of steatohepatitis, while only $10 \%$ of placebo were able to reach this target. This was the first study to show improvements in fibrosis without affecting steatohepatitis (48). Interestingly, there was a lack of significant improvement in fibrosis after 24 weeks of CVC, primarily because the placebo response increased significantly, whereas the antifibrotic effect was not increased further after 2 years of therapy compared to one. A 12-month, phase 3 study of CVC, AURORA (NCT03028740), in patients with NASH and stage 2-3 fibrosis is underway, with primary outcomes of biopsy improvement of fibrosis with no worsening of steatohepatitis, and superiority of CVC compared to placebo on the composite endpoint of cirrhosis, all-cause mortality and liver-related clinical outcomes.

\section{Granulocyte-colony stimulating factor (G-CSF)}

The innate immune system plays a crucial role in NAFLD and ALD, although each response is characterized and dominated by activation of specific subsets of cells. While monocyte and macrophage activation characterize NAFLD pathogenesis, making CCR2/5 a potential NASH therapeutic target, neutrophil activation is a key element in alcoholic steatohepatitis (in addition to macrophages). Neutrophil activation can create a proinflammatory milieu $(79,80)$, but simultaneously stimulate liver regeneration by mobilization of hematopoietic stem cells and also promote cellular repair (81-84). G-CSF purportedly improves survival in severe alcoholic hepatitis (85) by decreasing infection by improvement of immune paralysis (86). G-CSF has shown promise in treating patients with $\mathrm{SAH}$ who are non-responsive to current standard of care therapy with steroids (a Lille score $>0.45$ at day 7 ). A recent Phase 2 double blind placebo controlled study randomized 28 patients with histologically proven SAH, non-responsive to Prednisolone, to G-CSF (12 doses of $300 \mu \mathrm{g}$ in 28 days) or placebo. The 28-day mortality was similar in both groups (21.4\% in G-CSF, $28.6 \%$ in placebo; $\mathrm{P}=0.26$ ), but at 90 days there was a significant improvement in the MELD $(24.6 \pm 3.9$ to $19.4 \pm 3.7(\mathrm{P}=0.002))$ and lower 90 -day mortality $(35.7 \%$ versus $71.4 \% ; \mathrm{P}=0.04)$ in the G-CSF treated group. Receiving G-CSF (hazard ratio, 0.37; SD, 0.14-0.98; $\mathrm{P}=0.04$ ) and having a high baseline creatinine (hazard ratio, 4.12; SD, 1.7-10.3; $\mathrm{P}=0.002$ ) were predictive of 90 -day outcomes in steroid nonresponsive SAH. G-CSF was well tolerated. (NCT01820208) (56).

\section{S-adenosylmethionine}

S-adenosylmethionine is an antioxidant, the principle methyl donor involved in methylation reactions in the liver and crucial in glutathione (GSH) synthesis. Persons with ALD have decreased SAM levels; thus, elevating SAM levels could be a potential therapeutic target. Both animal (87) and human studies (88) have previously shown that increasing SAM levels improves liver injury by potentially restoring GSH synthesis, hence decreasing oxidative stress and hepatic stellate cell activation (89). In a phase 2 controlled trial, patients with ALD were randomized to 1.2 grams of SAM or placebo for 24 weeks. While there was an improvement in aminotransferases and hyperbilirubinemia, there was no significant improvement in clinical outcomes, biochemical parameters or histological features of ASH (55). The value of SAM in ALD remains unclear, and long-term clinical trials would be needed to establish its efficacy.

\section{Apoptosis signal-regulating kinase 1 inbibitor (ASK1i)}

Intracellular oxidative stress, especially from the endoplasmic reticulum, can activate ASK1 which in turns leads to phosphorylation of p38 mitogen-activated kinase and c-Jun N-terminal kinase (JNK) resulting in regulation of apoptotic and autophagic pathways which ultimately cause hepatic inflammation and myofibroblast activation resulting in fibrosis. (90). Hence, ASK1 Inhibition was an attractive target to prevent the progression of NASH, and an ASK1 inhibitor, selonsertib was evaluated. Despite preclinical data that the drug was effective in rodent models (91), and even in a phase 2 study when combined with a lysyl oxidase 
2 inhibitor simtuzamab (49), selonsertib showed no efficacy in two large phase 3 studies $(50,51)$ and is no longer being evaluated.

Selonsertib has also been investigated in severe alcoholic hepatitis in combination with prednisone compared to prednisone with placebo for 28 days in a phase 2 study (52). Analysis at 7 days showed no difference in Lille response score, and the 28 day response in MELD score, rate of infection and mortality were similar (52). There was a trend towards a higher rate of mortality in the selonsertib and prednisone group $(20.5 \%, 9 / 44)$ versus placebo and prednisone group $(6.1 \%, 3 / 49)$ that was not statistically significant $(\mathrm{P}=0.061)$ after 8 weeks on trial (52). Thus, there was no added benefit with selonsertib for the treatment of severe alcoholic hepatitis in combination with prednisone.

\section{Acetyl-coenzyme A carboxylase inhibition}

De novo lipogenesis (DNL) is increased in patients with NAFLD compared to both obese patients without NAFLD and to healthy controls. The increase in DNL and elevated peripheral fatty acids contribute to hepatic steatosis in NAFLD (92). Additionally, impaired fatty acid processing and trafficking can lead to lipotoxicity that manifests as inflammation, apoptosis, necrosis, and ballooning of hepatocytes (93). Acetyl-coenzyme A carboxylase (ACC) is the rate limiting step in DNL and has 2 distinct isoforms, ACC1 and ACC2, with ACC1 localized in the cytoplasm and ACC2 in mitochondria with both present in the liver (94). Inhibition of both ACC1 and ACC2 in murine models reduced hepatic steatosis, improved insulin sensitivity, reduced weight gain and had a favorable lipid profile (85). Thus, ACC inhibition is a logical target for steatosis and lipotoxicity for patients with NASH. GS-0976, firsocostat, a liver-targeted inhibitor of ACC1 and ACC2 has been used for the treatment of NASH in a pilot study that showed that after 12 weeks of firsocostat $20 \mathrm{mg}$, there was a decrease in DNL, steatosis based on MRI-PDFF and markers of liver injury (95). In a phase 2 study of firsocostat for 12 weeks in NASH patients with stage $1-3$ fibrosis, $48 \%$ of NAFLD patients receiving $20 \mathrm{mg}$ of firsocostat had a $\geq 30 \%$ relative decrease from baseline MRI-PDFF, $23 \%$ improved with firsocostat $5 \mathrm{mg}$ and $15 \%$ in the placebo group. The trial also demonstrated decreases in markers of fibrosis and liver chemistries (53). Additional studies have shown the added benefit from the combination of firsocostat and the FXR agonist cilofexor, leading to improvements in hepatic steatosis, liver stiffness on MRE, liver biochemistries and biomarkers of fibrosis in patients with NASH and stage 2-3 fibrosis treated for 12 weeks (96). Phase 2 studies using firsocostat in combination with the FXR agonist cilofexor in NASH are still ongoing (ATLAS, NCT03449446).

\section{Glucagon-like peptide-1 (GLP-1) analogues}

GLP-1 analogues have a well-established role in type 2 diabetes and obesity as they lead to weight loss, reduction of major cardiovascular events and improved glycemic control with fewer hypoglycemic events $(97,98)$. An initial pilot study in NASH in Japan evaluated 19 patients who failed lifestyle modifications and were given liraglutide $0.9 \mathrm{mg}$ injections daily for 24 weeks. Liraglutide was well tolerated in the subjects and liver biopsy was performed on 10 subjects who continued on liraglutide for 96 weeks, with improvements in histological inflammation (99). Further analysis was completed utilizing a small placebo-controlled study where $1.8 \mathrm{mg}$ injections of liraglutide were administered daily for 48 weeks with a histologic endpoint of definitive NASH resolution without worsening of fibrosis from baseline to end of treatment. There continued to be weight loss and better glycemic control in the group receiving liraglutide versus placebo. Histological improvements in ballooning and NASH resolution were demonstrated by liver biopsy in a modified intention-to-treat analysis. Thirty nine percent (9/23) of patients receiving liraglutide demonstrated NASH resolution with $61 \%(14 / 23)$ achieving improvements in hepatocyte ballooning versus $9 \%(2 / 22)$ and $32 \%(7 / 22)$ respectively with placebo (54). Further studies using GLP-1 drugs are underway utilizing a once weekly injection of semaglutide $2.4 \mathrm{mg}$ for 48 weeks in a phase 2 study, with fibrosis improvement on MRE as the primary endpoint and histological improvement as a secondary endpoint (NCT03987451). There are additional studies investigating semagluride for the treatment of NASH with varying daily dosing (NCT02970942) for 72 weeks with histologic endpoints. There are additional plans for a phase 2 combination study with GS-9674 (Cilofexor) and GS-0976 (Firsocostat) (NCT03987074).

\section{Conclusions}

In summary, the current climate for drug development to treat NASH and ALD is vibrant and promising. The sustained progress in elucidating the pathogenesis of these diseases continues to promote a highly interactive dynamic between basic, translational and clinical investigators, who 
are partnering with companies committed to developing new approaches to managing these illnesses. Hopefully affected patients and their caregivers will soon be the beneficiaries of this ongoing partnership through improved clinical outcomes, lifespan and quality of life.

\section{Acknowledgments}

Funding: None.

\section{Footnote}

Provenance and Peer Review: This article was commissioned by the Guest Editor (Ashwani K. Singal) for the series "Non-alcoholic Fatty Liver Disease and Alcoholic Liver Disease" published in Translational Gastroenterology and Hepatology. The article was sent for external peer review organized by the Guest Editor and the editorial office.

Conflicts of Interest: All authors have completed the ICMJE uniform disclosure form (available at http://dx.doi. org/10.21037/tgh.2020.01.04). The series "Non-alcoholic Fatty Liver Disease and Alcoholic Liver Disease" was commissioned by the editorial office without any funding or sponsorship. AD: Advisory Board: Intercept Pharmaceutical, Gilead Sciences; Speaker: Intercept Pharmaceutical; Consulting: Genfit, NASHNET. AA: Advisory Board: Intercept Pharmaceutical and Gilead Sciences Speaker: Intercept Pharmaceutical; Consulting: Genfit, Expert connect, Sterotherapeutics, Berenberg, NASHNET; Equity ownership: SanyalBio. SLF: Consultant to: 89 Bio, 3BV Bio, Axcella Health, Blade Therapeutics, Brii Biosciences, Bristol Myers Squibb, Can-Fite Biopharma, ChemomAb, Coherus, Escient Therapeutics, Forbion, Galmed, Genevant Genfit, Glycotest, Glympse Bio, Heparegenix, Janssen Pharmaceutical, Metacrine Inc., Morphic Therapeutics, North Sea Therapeutics, Novartis, Novo Nordisk, Pfizer Pharmaceuticals Scholar Rock, Second Genome, Surrozen, Viking Therapeutics; Stock options (all $<1 \%$ ): Akarna Therapeutics, BirdRock Bio, Blade Therapeutics, Conatus, DeuteRx, Escient, Exalenz, Galectin, Galmed, Genfit, Glympse, Indalo Therapeutics, Intercept, Lifemax. Metacrine, Nimbus, Northern Biologics, North Sea Therapeutics, Scholar Rock, Surrozen. The authors have no other conflicts of interest to declare.

Ethical Statement: The authors are accountable for all aspects of the work in ensuring that questions related to the accuracy or integrity of any part of the work are appropriately investigated and resolved.

Open Access Statement: This is an Open Access article distributed in accordance with the Creative Commons Attribution-NonCommercial-NoDerivs 4.0 International License (CC BY-NC-ND 4.0), which permits the noncommercial replication and distribution of the article with the strict proviso that no changes or edits are made and the original work is properly cited (including links to both the formal publication through the relevant DOI and the license). See: https://creativecommons.org/licenses/by-nc-nd/4.0/.

\section{References}

1. Estes C, Anstee QM, Teresa Arias-Loste M, et al. Modeling NAFLD Disease Burden in China, France, Germany, Italy, Japan, Spain, United Kingdom, and United States for the period 2016-2030. J Hepatol 2018;69:896-904.

2. Mellinger JL, Shedden K, Winder GS, et al. The high burden of alcoholic cirrhosis in privately insured persons in the United States. Hepatology 2018;68:872-82.

3. Tapper EB, Parikh ND. Mortality due to cirrhosis and liver cancer in the United States, 1999-2016: observational study. BMJ 2018;362:k2817.

4. Kim D, Li AA, Perumpail BJ, et al. Changing Trends in Etiology-Based and Ethnicity-Based Annual Mortality Rates of Cirrhosis and Hepatocellular Carcinoma in the United States. Hepatology 2019;69:1064-74.

5. Wong RJ, Cheung R, Ahmed A. Nonalcoholic steatohepatitis is the most rapidly growing indication for liver transplantation in patients with hepatocellular carcinoma in the U.S. Hepatology 2014;59:2188-95.

6. Shingina A, DeWitt PE, Dodge JL, et al. Future Trends in Demand for Liver Transplant: Birth Cohort Effects Among Patients With NASH and HCC. Transplantation 2019;103:140-8.

7. Tian C, Stokowski RP, Kershenobich D, et al. Variant in PNPLA3 is associated with alcoholic liver disease. Nat Genet 2010;42:21-3.

8. Romeo S, Kozlitina J, Xing C, et al. Genetic variation in PNPLA3 confers susceptibility to nonalcoholic fatty liver disease. Nat Genet 2008;40:1461-5.

9. Zhu L, Baker RD, Zhu R, et al. Gut microbiota produce alcohol and contribute to NAFLD. Gut 2016;65:1232.

10. Younossi ZM, Koenig AB, Abdelatif D, et al. Global epidemiology of nonalcoholic fatty liver disease-Meta- 
analytic assessment of prevalence, incidence, and outcomes. Hepatology 2016;64:73-84.

11. Younossi Z, Stepanova M, Ong JP, et al. Nonalcoholic Steatohepatitis Is the Fastest Growing Cause of Hepatocellular Carcinoma in Liver Transplant Candidates. Clin Gastroenterol Hepatol 2019;17:748-55.e3.

12. Cholankeril G, Wong RJ, Hu M, et al. Liver Transplantation for Nonalcoholic Steatohepatitis in the US: Temporal Trends and Outcomes. Dig Dis Sci 2017;62:2915-22.

13. Sanyal AJ, Harrison SA, Ratziu V, et al. The Natural History of Advanced Fibrosis due to Nonalcoholic Steatohepatitis: Data from the Simtuzumab Trials. Hepatology 2019;70:1913-27.

14. Dulai PS, Singh S, Patel J, et al. Increased risk of mortality by fibrosis stage in non-alcoholic fatty liver disease: Systematic Review and Meta-analysis. Hepatology. 2017;65:1557-65.

15. Hagstrom H, Nasr P, Ekstedt M, et al. Fibrosis stage but not NASH predicts mortality and time to development of severe liver disease in biopsy-proven NAFLD. J Hepatol 2017;67:1265-73.

16. Younossi ZM, Blissett D, Blissett R, et al. The economic and clinical burden of nonalcoholic fatty liver disease in the United States and Europe. Hepatology 2016;64:1577-86.

17. Yoon YH. Surveilance report \#105. Livert cirrhosis mortality in the United States: national,state and regional trends, 2000-2013. Alcoholism/NIAAA 2014. Available online: https://pubs.niaaa.nih.gov/publications/ surveillance105/Cirr13.pdf

18. Cholankeril G, Ahmed A. Alcoholic Liver Disease Replaces Hepatitis C Virus Infection as the Leading Indication for Liver Transplantation in the United States. Clin Gastroenterol Hepatol 2018;16:1356-8.

19. Wong T, Dang K, Ladhani S, et al. Prevalence of Alcoholic Fatty Liver Disease Among Adults in the United States, 2001-2016. JAMA 2019;321:1723-5.

20. Lackner C, Spindelboeck W, Haybaeck J, et al. Histological parameters and alcohol abstinence determine long-term prognosis in patients with alcoholic liver disease. J Hepatol 2017;66:610-8.

21. Louvet A, Labreuche J, Artru F, et al. Main drivers of outcome differ between short term and long term in severe alcoholic hepatitis: A prospective study. Hepatology 2017;66:1464-73.

22. Lee BP, Mehta N, Platt L, et al. Outcomes of Early Liver Transplantation for Patients With Severe Alcoholic
Hepatitis. Gastroenterology 2018;155:422-30.e1.

23. Puri P, Cholankeril G, Myint TY, et al. Early Liver Transplantation is a Viable Treatment Option in Severe Acute Alcoholic Hepatitis. Alcohol Alcohol 2018;53:716-8.

24. FDA. Noncirrhotic nonalcoholic steatohepatitis with liver fibrosis: developing drugs for treatment; guidance for instruction. FDA-2018-D-3632 2018.

25. FDA. Nonalcoholic steatohepatitis: developing drugs for treatment. Guidance for Industry 2019.

26. Harrison SA, Abdelmalek MF, Caldwell S, et al. Simtuzumab Is Ineffective for Patients With Bridging Fibrosis or Compensated Cirrhosis Caused by Nonalcoholic Steatohepatitis. Gastroenterology 2018;155:1140-53.

27. Han MAT, Altayar O, Hamdeh S, et al. Rates of and Factors Associated With Placebo Response in Trials of Pharmacotherapies for Nonalcoholic Steatohepatitis: Systematic Review and Meta-analysis. Clin Gastroenterol Hepatol 2019;17:616-29.e26.

28. Thursz MR, Richardson P, Allison M, et al. Prednisolone or pentoxifylline for alcoholic hepatitis. N Engl J Med 2015;372:1619-28.

29. Nguyen-Khac E, Thevenot T, Piquet MA, et al. Glucocorticoids plus N-acetylcysteine in severe alcoholic hepatitis. N Engl J Med 2011;365:1781-9.

30. Mathurin P, Louvet A, Duhamel A, et al. Prednisolone with vs without pentoxifylline and survival of patients with severe alcoholic hepatitis: a randomized clinical trial. JAMA 2013;310:1033-41.

31. Pavlov CS, Varganova DL, Casazza G, et al. Glucocorticosteroids for people with alcoholic hepatitis. Cochrane Database Syst Rev 2019;4:CD001511.

32. Ellenberg SS, Temple R. Placebo-controlled trials and active-control trials in the evaluation of new treatments. Part 2: practical issues and specific cases. Ann Intern Med 2000;133:464-70.

33. Temple R, Ellenberg SS. Placebo-controlled trials and active-control trials in the evaluation of new treatments. Part 1: ethical and scientific issues. Ann Intern Med 2000;133:455-63.

34. Ratziu V, Harrison SA, Francque S, et al. Elafibranor, an Agonist of the Peroxisome Proliferator-Activated Receptor-alpha and -delta, Induces Resolution of Nonalcoholic Steatohepatitis Without Fibrosis Worsening. Gastroenterology 2016;150:1147-59.e5.

35. Harrison S, Moussa S, Bashir M. MGL-3196, a selective thyroid hormone receptor-beta agonist significantly descreases hepatic fat in NASH patients at 12 weeks, the 
primary endpoint in a 36-week serial liver biopsy study. J Hepatology 2018;68:S38.

36. Loomba R, Neutel J, Bernard D. VK2809, a novel liverdirected thyrod receptor beta agonist, significantly reduces liver fat in patients with non-alcoholic fatty liver disease: a phase 2 randomized placebo-controlled trial. Hepatology 2018;68:1447A.

37. Mudaliar S, Henry RR, Sanyal AJ, et al. Efficacy and safety of the farnesoid $\mathrm{X}$ receptor agonist obeticholic acid in patients with type 2 diabetes and nonalcoholic fatty liver disease. Gastroenterology 2013;145:574-82.e1.

38. Neuschwander-Tetri BA, Loomba R, Sanyal AJ, et al. Farnesoid X nuclear receptor ligand obeticholic acid for non-cirrhotic, non-alcoholic steatohepatitis (FLINT): a multicentre, randomised, placebo-controlled trial. Lancet 2015;385:956-65.

39. Pockros PJ, Fuchs M, Freilich B, et al. CONTROL: a randomized phase 2 study of obeticholic acid and atorvastatin on lipoproteins in nonalcoholic steatohepatitis patients. Liver Int 2019;39:2082-93.

40. Younossi Z, Ratziu V, Loomba R, et al. GS-06-Positive Results from REGENERATE: A Phase 3 International, Randomized, Placebo-Controlled Study Evaluating Obeticholic Acid Treatment for NASH. J Hepatol 2019;70:e5.

41. Tully DC, Rucker PV, Chianelli D, et al. Discovery of Tropifexor (LJN452), a Highly Potent Non-bile Acid FXR Agonist for the Treatment of Cholestatic Liver Diseases and Nonalcoholic Steatohepatitis (NASH). J Med Chem 2017;60:9960-73.

42. Sanyal AJ, Lopez P, Lawitz E, et al. Tropifexor (TXR), an FXR Agonist for the Treatment of NASH-Interim Results from First Two Parts of Phase 2b Study Hepatology 2018;68:1444A-71A.

43. Sanyal A, Lopez P, Lawitz E, et al. SAT-357-Tropifexor, a farnesoid $\mathrm{X}$ receptor agonist for the treatment of nonalcoholic steatohepatitis: Interim results based on baseline body mass index from first two parts of Phase $2 \mathrm{~b}$ study FLIGHT-FXR. J Hepatol 2019;70:e796-e7.

44. Patel K, Harrison SA, Trotter JF, et al. The NonSteroidal FXR Agonist GS-9674 Leads to Significant Reductions in Hepatic Steatosis, Serum Bile Acids, and Liver Biochemistry in a Phase 2, Randomized, PlaceboControlled Trial of Patients with NASH. Hepatology 2018;68:438A.

45. Harrison SA, Rossi SJ, Paredes AH, et al. NGM282 Improves Liver Fibrosis and Histology in 12 Weeks in Patients With Nonalcoholic Steatohepatitis. Hepatology
2020;71:1198-212.

46. Sanyal A, Charles ED, Neuschwander-Tetri BA, et al. Pegbelfermin (BMS-986036), a PEGylated fibroblast growth factor 21 analogue, in patients with non-alcoholic steatohepatitis: a randomised, double-blind, placebocontrolled, phase 2a trial. Lancet 2018;392:2705-17.

47. Safadi R, Konikoff FM, Mahamid M, et al. The fatty acidbile acid conjugate Aramchol reduces liver fat content in patients with nonalcoholic fatty liver disease. Clin Gastroenterol Hepatol 2014;12:2085-91.e1.

48. Friedman SL, Ratziu V, Harrison SA, et al. A randomized, placebo-controlled trial of cenicriviroc for treatment of nonalcoholic steatohepatitis with fibrosis. Hepatology 2018;67:1754-67.

49. Loomba R, Lawitz E, Mantry PS, et al. The ASK1 inhibitor selonsertib in patients with nonalcoholic steatohepatitis: A randomized, phase 2 trial. Hepatology 2018;67:549-59.

50. Sciences G. Gilead Announces Topline Data From Phase 3 STELLAR-3 Study of Selonsertib in Bridging Fibrosis (F3) Due to Nonalcoholic Steatohepatitis (NASH). 2019. Available online: https://www.gilead.com/newsand-press/press-room/press-releases/2019/4/gileadannounces-topline-data-from-phase-3-stellar3-study-ofselonsertib-in-bridging-fibrosis-f3-due-to-nonalcoholicsteatohepatitis-nash

51. Sciences G. Gilead Announces Topline Data From Phase 3 STELLAR-4 Study of Selonsertib in Compensated Cirrhosis (F4) Due to Nonalcoholic Steatohepatitis (NASH), 2019. Available online: https://www.gilead. com/news-and-press/press-room/press-releases/2019/2/ gilead-announces-topline-data-from-phase-3-stellar4study-of-selonsertib-in-compensated-cirrhosis-f4-due-tononalcoholic-steatohepatitis-nash

52. Mathurin P, Dufour JF, Bzowej NH, et al. Selonsertib in Combination with Prednisolone for the Treatment of Severe Alcoholic Hepatitis: A Phase 2 Randomized Controlled Trial. Hepatology 2018;68:8A-9A.

53. Loomba R, Kayali Z, Noureddin M, et al. GS-0976 Reduces Hepatic Steatosis and Fibrosis Markers in Patients With Nonalcoholic Fatty Liver Disease. Gastroenterology 2018;155:1463-73.e6.

54. Armstrong MJ, Gaunt P, Aithal GP, et al. Liraglutide safety and efficacy in patients with non-alcoholic steatohepatitis (LEAN): a multicentre, double-blind, randomised, placebo-controlled phase 2 study. Lancet 2016;387:679-90.

55. Medici V, Virata MC, Peerson JM, et al. S-adenosyl-Lmethionine treatment for alcoholic liver disease: a double- 
blinded, randomized, placebo-controlled trial. Alcohol Clin Exp Res 2011;35:1960-5.

56. Shasthry SM, Sharma MK, Shasthry V, et al. Efficacy of Granulocyte Colony-stimulating Factor in the Management of Steroid-Nonresponsive Severe Alcoholic Hepatitis: A Double-Blind Randomized Controlled Trial. Hepatology 2019;70:802-11.

57. Pu S, Wu X, Yang X, et al. The Therapeutic Role of Xenobiotic Nuclear Receptors Against Metabolic Syndrome. Curr Drug Metab 2019;20:15-22.

58. Sanyal AJ, Chalasani N, Kowdley KV, et al. Pioglitazone, vitamin $\mathrm{E}$, or placebo for nonalcoholic steatohepatitis. $\mathrm{N}$ Engl J Med 2010;363:1185-6; author reply 1186.

59. Mahady SE, Webster AC, Walker S, et al. The role of thiazolidinediones in non-alcoholic steatohepatitis -a systematic review and meta analysis. J Hepatol 2011;55:1383-90.

60. Bril F, Kalavalapalli S, Clark VC, et al. Response to Pioglitazone in Patients With Nonalcoholic Steatohepatitis With vs Without Type 2 Diabetes. Clin Gastroenterol Hepatol 2018;16:558-66.e2.

61. Kim D, Kim W, Joo SK, et al. Subclinical Hypothyroidism and Low-Normal Thyroid Function Are Associated With Nonalcoholic Steatohepatitis and Fibrosis. Clin Gastroenterol Hepatol 2018;16:123-31.e1.

62. Pagadala MR, Zein CO, Dasarathy S, et al. Prevalence of hypothyroidism in nonalcoholic fatty liver disease. Dig Dis Sci 2012;57:528-34.

63. Liangpunsakul S, Chalasani N. Is hypothyroidism a risk factor for non-alcoholic steatohepatitis? J Clin Gastroenterol 2003;37:340-3.

64. Puliga E, Min Q, Tao J, et al. Thyroid Hormone Receptorbeta Agonist GC-1 Inhibits Met-beta-Catenin-Driven Hepatocellular Cancer. Am J Pathol 2017;187:2473-85.

65. Kelly MJ, Pietranico-Cole S, Larigan JD, et al. Discovery of 2-[3,5-dichloro-4-(5-isopropyl-6-oxo-1,6dihydropyridazin-3-yloxy)phenyl]-3,5-dio xo-2,3,4,5tetrahydro[1,2,4]triazine-6-carbonitrile (MGL-3196), a Highly Selective Thyroid Hormone Receptor beta agonist in clinical trials for the treatment of dyslipidemia. J Med Chem 2014;57:3912-23.

66. Taub R, Chiang E, Chabot-Blanchet M, et al. Lipid lowering in healthy volunteers treated with multiple doses of MGL-3196, a liver-targeted thyroid hormone receptorbeta agonist. Atherosclerosis 2013;230:373-80.

67. Sanyal AJ, Friedman SL, McCullough AJ, et al. Challenges and opportunities in drug and biomarker development for nonalcoholic steatohepatitis: findings and recommendations from an American Association for the Study of Liver Diseases-U.S. Food and Drug Administration Joint Workshop. Hepatology 2015;61:1392-405.

68. Harrison S, Guy CD, Bashir MR. In a placebo controlled 36 weeks phase 2 trial, treatment with MGL-3196 compared to placebo results in significant reductions of hepatic fat (MR-\{PDFF), liver enzymes, fibrosis biomarkers, atherogenic lipids and improvement in NASH on serial biopsy. Hepatology 2018.

69. Asgharpour A, Kumar D, Sanyal A. Bile acids: emerging role in management of liver diseases. Hepatol Int 2015;9:527-33.

70. Keitel V, Kubitz R, Haussinger D. Endocrine and paracrine role of bile acids. World J Gastroenterol 2008;14:5620-9.

71. Pellicciari R, Fiorucci S, Camaioni E, et al. 6alpha-ethylchenodeoxycholic acid (6-ECDCA), a potent and selective FXR agonist endowed with anticholestatic activity. J Med Chem 2002;45:3569-72.

72. Pellicciari R, Costantino G, Camaioni E, et al. Bile acid derivatives as ligands of the farnesoid $\mathrm{X}$ receptor. Synthesis, evaluation, and structure-activity relationship of a series of body and side chain modified analogues of chenodeoxycholic acid. J Med Chem 2004;47:4559-69.

73. Fiorucci S, Di Giorgio C, Distrutti E. Obeticholic Acid: An Update of Its Pharmacological Activities in Liver Disorders. Handb Exp Pharmacol 2019;256:283-95.

74. Trauner M, Gulamhusein A, Hameed B, et al. The Nonsteroidal Farnesoid X Receptor Agonist Cilofexor (GS-9674) Improves Markers of Cholestasis and Liver Injury in Patients With Primary Sclerosing Cholangitis. Hepatology 2019;70:788-801.

75. Schaap FG, Trauner M, Jansen PL. Bile acid receptors as targets for drug development. Nat Rev Gastroenterol Hepatol 2014;11:55-67.

76. Zhou M, Learned RM, Rossi SJ, et al. Engineered FGF19 eliminates bile acid toxicity and lipotoxicity leading to resolution of steatohepatitis and fibrosis in mice. Hepatol Commun 2017;1:1024-42.

77. Lee YA, Wallace MC, Friedman SL. Pathobiology of liver fibrosis: a translational success story. Gut 2015;64:830-41.

78. Lefebvre E, Moyle G, Reshef R, et al. Antifibrotic Effects of the Dual CCR2/CCR5 Antagonist Cenicriviroc in Animal Models of Liver and Kidney Fibrosis. PLoS One 2016;11:e0158156.

79. Ramaiah SK, Jaeschke H. Role of neutrophils in the pathogenesis of acute inflammatory liver injury. Toxicol 
Pathol 2007;35:757-66.

80. Taieb J, Delarche C, Paradis V, et al. Polymorphonuclear neutrophils are a source of hepatocyte growth factor in patients with severe alcoholic hepatitis. J Hepatol 2002;36:342-8.

81. Altamirano J, Miquel R, Katoonizadeh A, et al. A histologic scoring system for prognosis of patients with alcoholic hepatitis. Gastroenterology 2014;146:1231-9. e1-6.

82. Moreau R, Rautou PE. G-CSF therapy for severe alcoholic hepatitis: targeting liver regeneration or neutrophil function? Am J Gastroenterol 2014;109:1424-6.

83. Yannaki E, Athanasiou E, Xagorari A, et al. G-CSFprimed hematopoietic stem cells or G-CSF per se accelerate recovery and improve survival after liver injury, predominantly by promoting endogenous repair programs. Exp Hematol 2005;33:108-19.

84. Spahr L, Lambert JF, Rubbia-Brandt L, et al. Granulocytecolony stimulating factor induces proliferation of hepatic progenitors in alcoholic steatohepatitis: a randomized trial. Hepatology 2008;48:221-9.

85. Harriman G, Greenwood J, Bhat S, et al. Acetyl-CoA carboxylase inhibition by ND-630 reduces hepatic steatosis, improves insulin sensitivity, and modulates dyslipidemia in rats. Proc Natl Acad Sci U S A 2016;113:E1796-805.

86. Singh V, Sharma AK, Narasimhan RL, et al. Granulocyte colony-stimulating factor in severe alcoholic hepatitis: a randomized pilot study. Am J Gastroenterol 2014;109:1417-23.

87. Lieber CS. S-Adenosyl-L-methionine and alcoholic liver disease in animal models: implications for early intervention in human beings. Alcohol 2002;27:173-7.

88. Rambaldi A, Gluud C. S-adenosyl-L-methionine for alcoholic liver diseases. Cochrane Database Syst Rev 2006;(2):CD002235.

89. Karaa A, Thompson KJ, McKillop IH, et al. S-adenosylL-methionine attenuates oxidative stress and hepatic stellate cell activation in an ethanol-LPS-induced fibrotic rat model. Shock 2008;30:197-205.

90. Brenner C, Galluzzi L, Kepp O, et al. Decoding cell death

doi: $10.21037 / \operatorname{tgh} .2020 .01 .04$

Cite this article as: Asgharpour A, Dinani A, Friedman SL. Basic science to clinical trials in non-alcoholic fatty liver disease and alcohol-related liver disease: collaboration with industry. Transl Gastroenterol Hepatol 2021;6:5. signals in liver inflammation. J Hepatol 2013;59:583-94.

91. Budas G, Karnik S, Jonnson T, et al. Reduction of Liver Steatosis and Fibrosis with an Ask1 Inhibitor in a Murine Model of Nash is Accompanied by Improvements in Cholesterol, Bile Acid and Lipid Metabolism. J Hepatol 2016;64:S170.

92. Donnelly KL, Smith CI, Schwarzenberg SJ, et al. Sources of fatty acids stored in liver and secreted via lipoproteins in patients with nonalcoholic fatty liver disease. J Clin Invest 2005;115:1343-51.

93. Neuschwander-Tetri BA. Hepatic lipotoxicity and the pathogenesis of nonalcoholic steatohepatitis: the central role of nontriglyceride fatty acid metabolites. Hepatology 2010;52:774-88.

94. Kim KH. Regulation of mammalian acetyl-coenzyme A carboxylase. Annu Rev Nutr 1997;17:77-99.

95. Lawitz EJ, Coste A, Poordad F, et al. Acetyl-CoA Carboxylase Inhibitor GS-0976 for 12 Weeks Reduces Hepatic De Novo Lipogenesis and Steatosis in Patients With Nonalcoholic Steatohepatitis. Clin Gastroenterol Hepatol 2018;16:1983-91.e3.

96. Lawitz E, Gane E, Ruane P, et al. SAT-352-A combination of the ACC inhibitor GS-0976 and the nonsteroidal FXR agonist GS-9674 improves hepatic steatosis, biochemistry, and stiffness in patients with non-alcoholic steatohepatitis. J Hepatol 2019;70:e794.

97. 8. Pharmacologic Approaches to Glycemic Treatment: Standards of Medical Care in Diabetes-2018. Diabetes Care 2018;41:S73-85.

98. Eng C, Kramer CK, Zinman B, et al. Glucagonlike peptide-1 receptor agonist and basal insulin combination treatment for the management of type 2 diabetes: a systematic review and meta-analysis. Lancet 2014;384:2228-34.

99. Eguchi Y, Kitajima Y, Hyogo H, et al. Pilot study of liraglutide effects in non-alcoholic steatohepatitis and nonalcoholic fatty liver disease with glucose intolerance in Japanese patients (LEAN-J). Hepatol Res 2015;45:269-78. 